\title{
Calidad del cuidado de Enfermería brindado por profesionales en una ciudad colombiana
}

\author{
Quality of nursing care, provided by professionals, in a Colombian city \\ Qualidade do cuidado de enfermagem prestado por profissionais de uma cidade colombian \\ Eustorgio Jose Amed-Salazar* \\ Gloria Villareal-Amaris ** \\ Carmen Cecilia Alvis-Tous ***
}

\begin{abstract}
Autor de correspondencia
* $\triangle$ Enfermero, Mg Salud Pública, especialista en Gerencia de la Calidad y Auditoria en Salud. Docente Facultad Ciencias de la Salud, Universidad de Sucre. (Pregrado y posgrado) Correo: eustorgio.amed@unisucre.edu.co ORCID: https://orcid. org/0000-0002-6397-5732

${ }^{* * *}$ Enfermera Mg Salud Pública. Docente Facultad Ciencias de la Salud, Universidad de Sucre. (Pregrado y posgrado), Correo: gloria.villareal@unisucre.edu.co ORCID: https://orcid.org/0000-00019577-0160

${ }^{* * * *}$ Enfermera Especialista en Gerencia de la Educación. Docente programa Enfermería Universidad de Sucre. Correo: carmen.alviz@unisucre.edu.co. ORCID: https://orcid.org/0000-0002-6873-1462
\end{abstract}

\section{Resumen}

Objetivo: Evaluar la calidad del cuidado de Enfermería brindado por profesionales en instituciones de salud de Sincelejo (Colombia), mediante la aplicación del Care Q. Materiales y métodos: Estudio descriptivo y transversal. La población se conformó por 515 pacientes, se estimó un cálculo de muestra inicial de 212 pacientes quienes respondieron al instrumento; tras eliminación por filtro a través de la pregunta control quedaron finalmente 123 pacientes que fueron captados mediante el tipo de muestreo no probabilístico de acuerdo con las condiciones de la investigación. La calidad fue valorada a través del instrumento Care Q que evalúa las siguientes dimensiones: accesibilidad, explica, facilita, conforta, se anticipa, mantiene relaciones de confianza, monitorea y hace seguimiento. Se utilizaron: el análisis de frecuencias, porcentajes con su intervalo de confianza y alfa de Cronbach para la comprobación de fiabilidad del instrumento "Care Q" al contexto. Resultados: Se pudo hallar una calidad total alcanzada en un $62 \%$. La dimensión con más alta calidad fue "monitorea y hace seguimiento" ( $73,2 \%)$ y la más baja "se anticipa" $(56,1 \%) .42 \%$ de los pacientes dijeron no haber sido atendidos y no reconocen a la Enfermera (o) del servicio. Conclusión: la calidad global del cuidado de Enfermería percibido por el usuario en las IPS estudiadas es regular (62\%), con amplias oportunidades de mejora, lo que merece una revisión desde las instituciones de salud, las Enfermeras (os) y la academia.

Palabras clave: Atención de Enfermería, calidad de la atención de salud, evaluación en Enfermería. 
was evaluated through the Care Q instrument, which evaluates the following dimensions: accessibility, explains, facilitates, comforts, anticipates, maintains trusting relationships, monitors and follows up. Frequency analysis, percentages with their confidence interval and the Cronbach's alpha were used to verify the reliability of the instrument "Care Q" to the context. Results: The reached quality found was $62 \%$. The dimension with the highest quality was "monitoring and follow up" $(73.2 \%)$ and the lowest "anticipates" (56.1\%). 42\% of the patients said they were unattended and do not recognize the nurse. Conclusion: The global quality of nursing care perceived by the users in the studied IPS (Healthcare Provider) is regular (62\%), with ample opportunities of improving, which merits a review from the health institutions, nurses and the academy.

Keywords: Nursing care, health care quality, nursing evaluation.

\begin{abstract}
Resumo
Objetivo: Avaliar a qualidade dos cuidados de enfermagem oferecido por profissionais em instituições hospitalares de Sincelejo (Colômbia), mediante a aplicação do Care Q. Materiais e métodos: Estudo descritivo transversal. A população foi de 515 pacientes e estimou-se uma amostra inicial de 212 que responderam a escala. Após a realização do filtro utilizando-se uma pergunta controle, foram analisados 123 questionários mediante amostragem não probabilística de acordo com as condições da pesquisa. O Care Q avalia a qualidade as dimensões: acessibilidade, explicação, facilidade, conforto, antecipação, relacionamento de confiança, monitoramento e acompanhamento. Utilizou-se a análise de frequência, percentagens com intervalos de confiança e alfa de Cronbach para a comprobação de fiabilidade da escala Care Q para o contexto. Resultados: Encontrou-se uma qualidade total de $62 \%$. A dimensão com maior qualidade foi "monitoramento e acompanhamento" (73.2\%) e a menor qualidade observou-se na antecipação (56.1\%). $42 \%$ dos participantes referiram não ter recebido atendimento e não reconheceram a enfermeira (o) do serviço. Conclusão: A qualidade global dos cuidados de enfermagem percebidos pelos usuários das instituições hospitalares estudadas é moderada (62\%), com amplias oportunidades de melhoramento, o que merece atenção pelas instituições de saúde, as enfermeiras (os) e as escolas de enfermagem.
\end{abstract}

Palavras-Chave: Cuidados de enfermagem, garantia da qualidade dos cuidados de saúde, avaliação de desempenho profissional, enfermagem

\section{Introducción}

La estructura actual del Sistema General de Seguridad Social en Salud colombiano es proclive a la búsqueda activa de la calidad; por tanto provee y facilita el uso de herramientas de valoración, seguimiento y auditoria desde el Sistema Obligatorio de Garantía de la Calidad (1). De ahí, que se pueda escuchar la opinión del usuario colocando como elemento relevante la evaluación de calidad en la atención en salud, concepto en el que se ha tenido un interés creciente en los últimos años. Dicha evaluación tiene como centro de acción al paciente, de allí la importancia por valorar su satisfacción, de estimar la calidad del cuidado que se le brinda, de usar medidas organizacionales de calidad que de manera particular se hacen desde el rol de Enfermería y que articuladas con la política sanitaria del país, pueden ayudar a mostrar cómo la atención de las Enfermeras(os) mejora la calidad, la seguridad y la atención de la salud en general $(2,3)$.
Valorar la calidad de los servicios de salud se ha transformado en una necesidad progresiva en la actualidad, con la intención de brindar mejores servicios. Este concepto se ha definido de muchas formas, la propuesta de Donabedian ha sido una de las más aceptadas; sin embargo, no hay una definición universal y depende mucho de la percepción de quien la defina, influyendo notablemente el rol que éste desempeñe. El concepto de calidad para el médico, el paciente, el administrador, el político, el empleado, el empresario, no es el mismo y cada quien valora de forma diferente sus componentes; es decir, que el significado de la calidad surge por las múltiples experiencias de los usuarios al recurrir a los servicios de salud. Es por eso, que en ese concepto de calidad en salud está la calidad del cuidado como parte de la mejora en la atención sanitaria y como principio legal y normativo de la profesión de Enfermería en el país (4-7). 
ISSN-PRINT

1794-9831

E-ISSN 2322-7028

Vol. 16 No. 2

May - Ago 2019

Cúcuta, Colombia
Por su parte, los pacientes tienen una autoconcepción de la atención que reciben; es por eso, que para satisfacer sus expectativas se necesita conocer cuáles son los componentes de esa concepción de atención. Al desconocer lo que piensan puede traducirse en "no conformidad" convirtiéndose en usuarios insatisfechos y para satisfacerlos no se pueden escatimar esfuerzos. Además, es parte de la naturaleza disciplinar de Enfermería garantizar cuidados con calidad a los pacientes (8); por ello, se debe brindar una atención de calidad en todos los aspectos, solo así se logra que la salud sea una realidad concreta (9). Y si a esto se suman los atributos interpersonales y transpersonales de coherencia, empatía y calidez en el cuidado descritos por Jean Watson en su teoría de cuidado humano (10), se puede identificar un constructo que merece valorarse sistemáticamente.

Hay que considerar, la necesidad de evaluar continua y seguidamente el comportamiento del cuidado brindado, con el objeto de identificar los puntos susceptibles de intervención temprana; y desde allí identificar las probables alteraciones del cuidado, examinando los vacíos en la atención y optimizando los procesos asistenciales. Para lograrlo, se pueden generar mejoras continuas, no solo en el cuidado del paciente, sino que también se debe poner atención al proceso de atención en general; acciones que permitirán estar en permanente desarrollo profesional, fortalecer actitudes y valores que faciliten la humanización en su quehacer cotidiano (11) y mejorar la calidad de la atención de Enfermería, que es el producto combinado de la expectativa y percepción del paciente. El equipo de salud, especialmente la Enfermera, debe conocer lo que perciben los pacientes de la atención de Enfermería y qué factores influyen en él $(12,13)$.

Se debe precisar, que cuidar es parte esencial del quehacer Enfermero, se reconoce como una forma de diálogo que acarrea ir más allá de la observación y de la reflexión; involucra modelar, comunicar, confirmar y practicar; es una forma de relación y crecimiento mutuo. También, requiere tener en cuenta sentimientos, habilidades de conexión, participación y conocimiento. Igualmente, implica una relación de dos sujetos que son iguales como seres humanos. Es por tanto, un proceso dinámico que incluye el entorno de quien se cuida y en él la Enfermera (o) quien debe ser evaluada(o) en su capacidad como cuidador(a) (14).
Desde esta óptica se puede entender un cuidado centrado en la persona, quien en lugar de estar inmersa en una situación patológica, vive experiencias de salud relacionadas con su entorno. Vinculada con este enfoque la práctica de la Enfermería se basa en la instrumentación de un cuidado contextualizado, orientado hacia lo que se realiza con la persona o en nombre de ella, y no tanto en lo que se le realiza, es decir, que el cuidado debe ser como una construcción co-creada. Se parte de considerar la calidad de cuidado de la Enfermería como un complejo sistema de componentes, desde la óptica holística, que buscan satisfacer las necesidades del cuidado de los usuarios en medio de la relación entre enfermera y paciente; esos componentes están definidos en seis dimensiones de cuidados expuestas por P. Larson $(15,16)$ :

Accesibilidad: alude a comportamientos de cuidado que son esenciales en la relación de apoyo y ayuda administrada de forma oportuna, como es el acercarse al paciente dedicándole tiempo.

Explica y facilita: son los cuidados que brinda el profesional de la Enfermería para dar a conocer aspectos que para el usuario son desconocidos o difíciles de entender en relación a su enfermedad, tratamiento o recuperación; del mismo modo, ofrece información requerida en forma clara, haciendo fácil o posible la ejecución de los cuidados para su bienestar o recuperación.

Conforta: Se refiere a los cuidados que ofrece la Enfermera con el fin de que el usuario, la familia y los allegados se sientan cómodos, infundiéndoles ánimo $\mathrm{y}$ vigor, en un entorno que favorezca el bienestar.

Se anticipa: Valora los cuidados que los y las Enfermeros/as planean con anterioridad teniendo en cuenta las necesidades del usuario, con el fin de prevenir complicaciones.

Mantiene una relación de confianza: Son los cuidados que ofrecen los profesionales de enfermería para que el usuario hospitalizado tenga empatía con ellos. Estos cuidados van dirigidos a la recuperación, haciendo sentir al enfermo como persona única, confiada, serena y segura. Tienen que ver con la cercanía y la presencia física del personal de enfermería hacia el usuario.

Monitorea y hace seguimiento: referido a los cuidados de enfermería que implican un conocimiento propio 
de cada usuario y dominio de lo científico técnico y de los procedimientos que realiza. Incluye los planes y acciones que son realizados para enseñar a su grupo de Enfermería la forma como se deben realizar los procedimientos y mantener la observación para que las acciones de cuidado se hagan bien y a tiempo (16).

Atendiendo a las consideraciones anteriores, es importante realizar una exploración de la calidad del cuidado de Enfermería en Sincelejo. Para lograr esta meta, se tendrán en cuenta los resultados valiosos que ofrecen el sistema de salud en general y las IPS en particular, en lo que se refiere a la mejora en la calidad de la atención en salud; asimismo, se utilizaran de la mejor manera posible las competencias del profesional de Enfermería, lo que enaltece la profesión además de generar garantía de satisfacción a los pacientes.

\section{Objetivos}

\section{Objetivo General}

Evaluar la calidad del cuidado de Enfermería brindado por profesionales en instituciones de salud de Sincelejo, mediante la aplicación del Care Q.

\section{Objetivo específico}

Describir la percepción de los comportamientos del cuidado de Enfermería en las categorías: accesible, explica, facilita, anticipa, conforta y mantiene relaciones de confianza.

\section{Materiales y métodos}

Estudio de diseño descriptivo y transversal. La población se conformó por 515 personas que correspondía al número esperado de pacientes, según las camas habilitadas de dos Instituciones Prestadoras de Servicios de Salud (IPS) que hacen parte del convenio docencia servicio con la Universidad de Sucre, en la ciudad de Sincelejo. Se ejecutó entre el segundo semestre de 2017 y el primer semestre de 2018. Para todos los cálculos muestrales necesarios se utilizó la calculadora epidemiológica OpenEpi versión 3.0.1, obteniéndose una muestra de 221 pacientes, con un margen de error del 5\%, una confiabilidad del 95\% y una frecuencia (p) de 50\%, sin reemplazo. Se aplicó una técnica de muestreo no probabilística a criterio, dado que el participante debía responder a la pregunta control; se completó teniendo en cuenta el tiempo asignado a la fase planificada para la recolección de la información y en el periodo definido por las IPS participantes. De acuerdo al número de pacientes hospitalizados al momento del estudio, a los criterios de exclusión, incluida la pregunta control en la que se indagó si conocían o habían tenido contacto con la Enfermera, finalmente quedó una población accesible de 123 pacientes, cuyos nombres fueron verificados por la lista de hospitalizados y por último se les aplicó el instrumento "Care Q" de Patricia Larson.

Dicho instrumento, fue validado para la población Colombiana por Sepúlveda y otros, en el Hospital Universitario Clínica San Rafael de Bogotá en 2009. Consta de 50 ítems, valorado mediante escala tipo Likert de: 1 a 5, nunca, a veces, casi siempre, siempre; con una fiabilidad valorada con alfa de Cronbach $=0,8(16)$

También se hizo una prueba piloto en una IPS ubicada fuera de la ciudad, con características similares a las IPS del estudio, para probar la metodología e instrumentos de evaluación, así como su fiabilidad, aplicando dicho análisis mediante modelo Alfa de Cronbach, obteniéndose un estadístico global de la escala con valor de 0,9 .

De igual modo, se estimó para esta institución una población de referencia de 30 camas hospitalarias disponibles, habilitadas para la atención, estudiadas en su totalidad, dado que la población era pequeña. En este sentido, el cálculo muestral se estimó con un margen de error del 5\%, una confiabilidad del 95\% y una frecuencia (p) de $50 \%$, para un total de 28 pacientes a estudiar, lo cual no ofrece una diferencia significativa.

Por otra parte, la recolección de la información se realizó mediante dispositivos móviles, tipo Tablets, interconectadas a través de red Wifi (o datos) con el fin de alimentar una base de datos única simultánea, con almacenamiento en tiempo real en un servidor virtual con acceso restringido del usuario. Con este procedimiento se buscó seguridad de datos, eficiente gestión de información y oportuna disponibilidad, evitando reprocesos y errores en la etapa de tabulación. La base de datos obtenida se exportó en formato *.xls para su procesamiento en el paquete estadístico IBM SPSS Statistics versión 21. Respecto al análisis descriptivo los datos se expresaron en frecuencias, porcentajes, promedios y desviación estándar 
ISSN-PRINT

1794-9831

E-ISSN 2322-7028

Vol. 16 No. 2

May - Ago 2019

Cúcuta, Colombia de los comportamientos del cuidado de Enfermería, teniendo en cuenta las categorías: accesible, explica, facilita, anticipa, conforta y mantiene relaciones de confianza; que posteriormente fueron evaluadas por el instrumento.

Debe señalarse, que estos estimadores se valoraron específicamente por sexo, edad y escolaridad, con el objeto de hacer control Counfunding (variables de confusión). Para la estimación de la métrica de calidad numérica se realizó una ruta matemática que permitió transformar la variable a escala de 1 a 100, luego se asignaron pesos porcentuales que permitieron construir una variable total conjunta que estimó la Calidad del cuidado. Sin embargo, se observaron variables de confusión que dependieron del estado de ingreso del sujeto, nivel de dolor, situación de angustia al ingreso; cambios que no se pueden controlar, y que por tanto no pueden ser contemplados en este estudio.

Los criterios de inclusión que se tuvieron en cuenta fueron los siguientes: pacientes que se encontraran hospitalizados en el momento de realizar la encuesta, mayores de 18 años, con más de tres días de estancia hospitalaria y que desearan voluntariamente contestar la encuesta.

Igualmente, como criterios de exclusión se consideraron los siguientes: no haber sido atendidos por un profesional de enfermería del servicio, pacientes que presenten alteraciones mentales, que no tuvieran la voluntad de contestar el instrumento, que no supieran leer y/o escribir y que carecieran de la ayuda de un cuidador.

En cuanto a la selección de los participantes, se solicitó una lista de los pacientes hospitalizados en el servicio, se verificó cuáles tenían un mínimo de 72 horas de hospitalización, se acudió al área asignada a cada uno de ellos, se abordaron en un horario y situación que no afectara la tranquilidad del paciente y se les solicitó su participación voluntaria.

Esta investigación se enmarcó en la Declaración Mundial de Helsinki enmendada en la $64^{\mathrm{a}}$ Asamblea general en Fortaleza, Brasil 2013 (17). Se rigió por lo contemplado en la Resolución 008430 de 1993 del Ministerio de Protección Social (18). Se obtuvo el aval del Comité de Ética de la Universidad de Sucre: acta 04-2018.

Respecto a la recolección de la información, previamente se diligenció el consentimiento informado a los pacientes, mediante el cual se hizo la declaratoria de participar en la investigación en forma voluntaria. También se les garantizó el anonimato en sus respuestas, explicándoles previamente que los datos obtenidos serían utilizados para la descripción de la calidad del cuidado de Enfermería brindado por profesionales y por tanto la información sería de carácter confidencial y privada. Estas actividades fueron realizadas por auxiliares de investigación, quienes fueron capacitadas por los investigadores en el uso y manejo del cuestionario Care Q y demás formatos del estudio.

\section{Resultados}

A partir de su experiencia de cuidado, los pacientes procedieron a manifestar su percepción. La valoración de las categorías o dimensiones que componen la calidad del cuidado, según Patricia Larson, permitió observar que entre $22 \%$ y $39 \%$ de los participantes percibe "poca o ninguna" participación de la Enfermera (o) en el cumplimiento de las dimensiones del cuidado estudiadas (Tabla 1).

Es importante resaltar que, en promedio, las personas que reconocen a la Enfermera(o) y han sido sujetos de su cuidado, consideran significativamente (78\%) que estos profesionales les hacen monitoreo y seguimiento permanente. En general para esta dimensión se alcanzó una calidad del $73,2 \%$, tomando en cuenta el puntaje máximo logrado, según la sumatoria de los ítems (Tabla 2); lo cual la posiciona como la categoría en la que mayor valoración de calidad se obtuvo.

Es importante señalar, que el puntaje de la dimensión anterior está bastante parejo en relación con las dimensiones: accesibilidad, explica y facilita, conforta, y mantiene relaciones de confianza; en este sentido el paciente piensa que las cumplen o llevan a cabo "parcial o totalmente", entre $70 \%$ y $73 \%$ (Tabla 1). En el análisis global de calidad se pudo encontrar un comportamiento sobre estas dimensiones que oscila entre el $60 \%$ y $61,8 \%$ (tabla 2), cifras bastante regulares con respecto al alcance de calidad de cuidado de Enfermería.

La dimensión "se anticipa" muestra el comportamiento más bajo de las categorías estudiadas, solo un poco más de la mitad (61\%) de los pacientes consideran que la Enfermera(o) lo hace parcial o totalmente (Tabla1) y en lo referente a la valoración global de calidad se obtuvo un $56,1 \%$ de calidad alcanzada (Tabla 2) 
Tabla 1. Promedio Situación de las dimensiones de la calidad de cuidado de Enfermería.

\begin{tabular}{lcccccc}
\hline \multicolumn{1}{c}{ DIMENSION } & $\begin{array}{c}\text { Fr } \\
\text { media }\end{array}$ & $\begin{array}{c}\text { POCA O } \\
\text { NINGUNA }\end{array}$ & IC 95\% & $\begin{array}{c}\text { Fr } \\
\text { media }\end{array}$ & $\begin{array}{c}\text { PARCIAL O } \\
\text { TOTAL }\end{array}$ & IC 95\% \\
\hline ACCESIBILIDAD & 37 & $30 \%$ & $21,5-38,5$ & 86 & $70 \%$ & $61,4-78,4$ \\
EXPLICA FACILITA & 36 & $29 \%$ & $20,8-37,7$ & 87 & $71 \%$ & $62,2-79,1$ \\
CONFORTA & 36 & $29 \%$ & $20,8-37,7$ & 87 & $71 \%$ & $62,2-79,1$ \\
SE ANTICIPA & 47 & $39 \%$ & $29,2-47,2$ & 76 & $61 \%$ & $52,7-70,7$ \\
MANTIENE RELACIONES DE CONFIANZA & 34 & $27 \%$ & $19,3-35,9$ & 89 & $73 \%$ & $64,0-80,6$ \\
MONITOREA HACE SEGUIMIENTO & 27 & $22 \%$ & $24,2-29,6$ & 96 & $78 \%$ & $70,3-85,7$ \\
\hline
\end{tabular}

E-ISSN 2322-7028

Vol. 16 No. 2

May - Ago 2019

Cúcuta, Colombia

Fuente: construcción de los autores.

Teniendo en cuenta la pregunta filtro donde se indagó si conocían o habían tenido contacto con la (el) Enfermera (o), los pacientes mostraron su "no conformidad" al manifestar que no tienen la posibilidad de acceder a este profesional desde el inicio de la atención; este hecho se refleja en que una proporción importante, el (42\%) no los conocen o identifican.

Se encontró de manera específica para la dimensión "accesibilidad" que más de la mitad (52\%) de los pa- cientes manifiesta que la (el) Enfermera (o) siempre les pide que la(o) llamen si necesitan de ella (el); aproximadamente, la mitad de las personas (48\%) cree que la Enfermera (o) casi siempre responde rápidamente a su llamado (Grafico 1). Es importante mencionar que cerca de un tercio $(32,5 \%)$ manifiesta que la Enfermera (o) le da los medicamentos y realiza los procedimientos a tiempo; sin embargo, consideran que esto ocurre solo a veces, mientras que poco más de un cuarto $(25,2 \%)$ informa que la Enfermera (o) nunca lo hace (Figura 1).

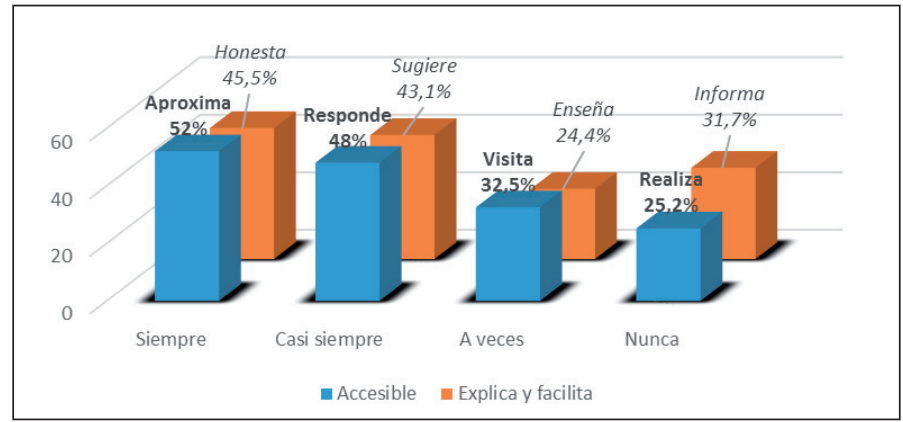

Figura 1: Items relevantes de categorías: Accesible y explicafacilita

Fuente: construcción de los autores

Para la dimensión "explica y facilita" se pudo encontrar que un número cercano a la mitad $(48,8 \%)$ de los pacientes informa que la Enfermera (o) siempre les enseña cómo cuidar de sí mismo; 43,1 \% de los participantes consideran que la Enfermera (o) casi siempre les sugiere preguntas que pueden formularle a su Médico cuando lo necesiten; el 31,7 $\%$ precisa que ella (el) nunca le informa sobre los grupos de ayuda para el control y seguimiento de su enfermedad (Figura 1).
En la dimensión "conforta” se observó que la mayoría de pacientes $(60,2 \%)$ expresa que la Enfermera (o) siempre es amable con ellos a pesar de tener situaciones difíciles; un número cercano a la mitad $(48,1 \%)$ considera que casi siempre la (el) Enfermera (o) se esfuerza para que ellos puedan descansar cómodamente; un número importante de participantes $(47,1 \%)$ reporta que solo a veces la (el) Enfermera (o) se sienta con ellos para entablar una conversación y el $9,3 \%$ indica que nunca establece contacto físico con ella (el) cuando necesita consuelo (Figura 2). 
ISSN-PRINT

$1794-9831$

E-ISSN 2322-7028

Vol. 16 No. 2

May - Ago 2019

Cúcuta, Colombia

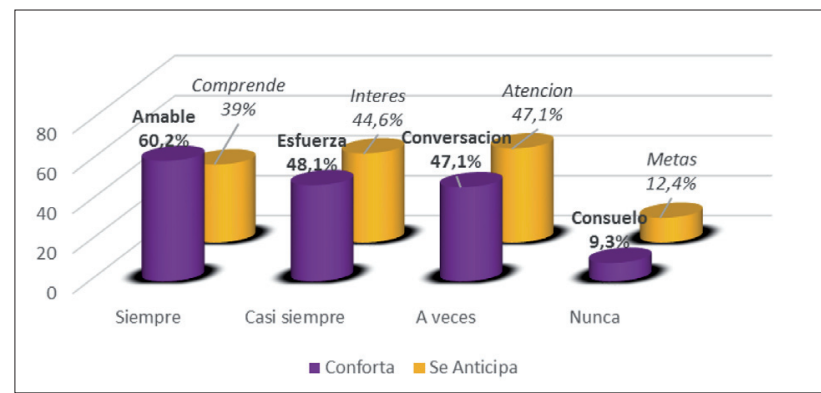

Figura 2: Items relevantes de categorías: Confort y se anticipa

Fuente: construcción de los autores.

Se identificó en la dimensión "se anticipa" que un número significativo de pacientes (39\%) cree que la Enfermera siempre comprende que la experiencia que viven es difícil para ellos y les presta especial atención durante este tiempo; un gran porcentaje $(44,6 \%)$ de los participantes reconoce que casi siempre ésta continúa interesada en ellos aunque hayan pasado por una crisis o fase crítica; muchos de los pacientes $(47,1 \%)$ consideran que solo a veces la Enfermera les presta mayor atención durante la noche; y el 12,4\% manifiesta que nunca le ayuda a mantener metas razonables. (Figura 2)
De igual forma, pudo encontrarse como hallazgo en la dimensión "mantiene relaciones de confianza" que más de la mitad de los pacientes $(52,9 \%)$ coinciden en que la (el) Enfermera (o) mantiene siempre un acercamiento respetuoso con ellos; el 47,9\% expresa que casi siempre le permite expresar totalmente sus sentimientos sobre su enfermedad y tratamiento; mientras que el $38,9 \%$ indica que solo a veces le ayuda a aclarar sus dudas en relación a su situación y 8,3 \% manifiestan que nunca los anima a formularle preguntas a su médico relacionadas con su situación de salud (Figura 3 ).

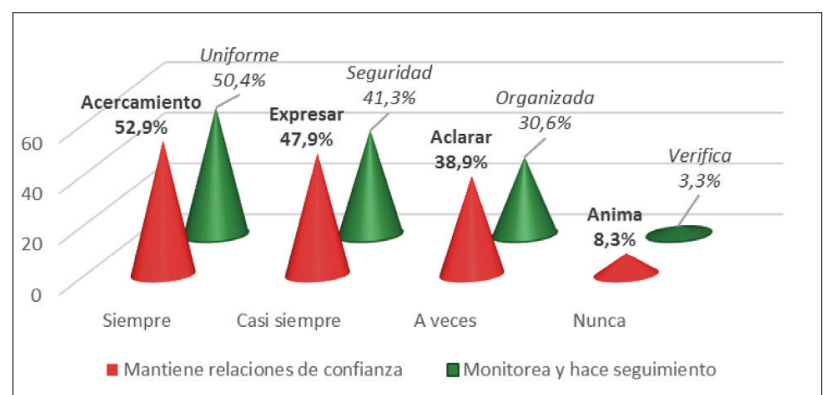

Figura 3: Items relevantes de categorías: Mantiene relaciones de confianza y monitorea - hace seguimiento.

Fuente: construcción de los autores.

En cuanto a la dimensión "monitorea y hace seguimiento" - la mejor calificada- el hallazgo estuvo en que la mayoría de los pacientes $(50,4 \%)$ consideran que siempre el uniforme y carnet que porta la Enfermera (o) la (o) caracteriza; un porcentaje importante $(41,3 \%)$ señala que casi siempre la (el) Enfermera (o) realiza los procedimientos con seguridad; mientras que el 30,6\% indica que solo a veces es organizada (o) en la realización de su trabajo; un pequeño grupo de pacientes $(3,3 \%)$ manifestó que nunca se asegura de la hora establecida para los procedimientos especiales (Figura 3 ).

Teniendo en cuenta los resultados obtenidos se procedió a calcular la métrica de calidad del cuidado de Enfermería. Esta medida de calidad se obtuvo con la sumatoria general de las 6 dimensiones, obteniéndose un puntaje de 17.167 , de 27.675 puntos posibles, para un total de $62 \%$ de alcance de calidad global; es decir, que se desea que exista una mejora de la calidad del cuidado de Enfermería del $38 \%$. Desde este punto de vista, se encuentra como hallazgo de gran importancia para el mejoramiento continuo de la práctica profesional, que poco más de un tercio (38\%) de los pacientes estudiados sugiere que se mejore la calidad de los cuidados ofrecidos por parte del profesional de la Enfermería (Tabla 2). 
Tabla 2. Proporción Calidad de Cuidado de Enfermería por dimensión.

\begin{tabular}{|c|c|c|c|}
\hline DIMENSION & Puntos alcanzados & $\begin{array}{c}\text { Puntos máximo } \\
\text { posible }\end{array}$ & $\%$ Calidad Alcanzado \\
\hline ACCESIBLE & 1851 & 3075 & 60,0 \\
\hline EXPLICA FACILITA & 1878 & 3075 & 61,0 \\
\hline CONFORTA & 3407 & 5535 & 61,5 \\
\hline SE ANTICIPA & 3454 & 6150 & 56,1 \\
\hline MANTIENE RELACIONES DE CONFIANZA & 3424 & 5535 & 61,8 \\
\hline MONITOREA HACE SEGUIMIENTO & 3153 & 4305 & 73,2 \\
\hline Total & 17167 & 27675 & 62,0 \\
\hline$\%$ Susceptible de mejora & - & - & 38,0 \\
\hline
\end{tabular}

Fuente: construcción de los autores.

Con respecto al resultado de la fiabilidad del instrumento Care Q, fue un Alfa de Cronbach de 0,9 indicando un excelente comportamiento para su aplicación (19). Al evaluar la calidad del cuidado de Enfermería, brindado por profesionales en instituciones de salud de la ciudad de Sincelejo, se encontró que un número importante $(42 \%)$ de los pacientes dijeron no haber sido atendidos y no reconocen a la Enfermera (o) del servicio donde se encuentran, dentro de la entidad hospitalaria.

\section{Discusión}

El presente estudio indagó con respecto a la calidad del cuidado de Enfermería, a través de la percepción del paciente, teniendo en cuenta las dimensiones de valoración propuestas por Patricia Larson, planteando una métrica en atención a tales dimensiones. En este sentido, la calidad total alcanzada fue de $62 \%$, una valoración bastante "regular", superando apenas la mitad, situación contrastable con los hallazgos de Lenis et al. (20) quienes encontraron en su estudio más de un $70 \%$ de satisfacción global del cuidado en Enfermería, percibida como alta; igualmente, Torres y Buitrago (21) en su investigación identificaron que 98,7\% (sumando todos los ítems positivos) de los pacientes valoraron positivamente la calidad de atención de estos profesionales.

Es decir, que el presente estudio encuentra una percepción de calidad baja por parte de los pacientes, en comparación con otros análisis realizados en ciudades distintas; por tanto se evidencia una realidad que se aleja de una expectativa de calidad desde la percepción del paciente, mediada por las dimensiones analizadas y por un contexto laboral adverso a la ejecución del cuidado del Enfermero; factores que tienen como resultado un servicio de salud deshumanizado (22) y un liderazgo de la Enfermería ineficaz en la mejora de la calidad de atención al paciente $(23,24)$.

Por otra parte, el hallazgo de un 38\% de susceptibilidad en la mejora de calidad del cuidado de Enfermería desde la percepción del paciente, coincide con los estudios de Molina et al. (25) quienes concluyen que está influida por la práctica, los recursos y en alto grado por la satisfacción de los pacientes con la asistencia recibida; lo que supone, que la intervención de varios actores del escenario de la salud (academia, prestadores, administradores) podría contribuir en la mejora integral del cuidado.

La calidad de cuidados de la Enfermería necesita no solo un análisis global o general sino que, según el modelo de evaluación Care Q, se debe realizar una valoración por dimensiones de ese cuidado. Siguiendo este proceso se pudo demostrar que en el monitoreo y seguimiento al cuidado del paciente una importante proporción de las personas (78\%) consideran que la (el) Enfermera (o) lo hace de la mejor manera y con calidad; es decir, que éste comportamiento se corresponde con lo expuesto por Guzmán (26) quien reporta que el $88,3 \%$ de sus participantes lo consideran en un alto nivel de satisfacción frente a la calidad de la atención recibida. Como se puede percibir, es un hallazgo alentador que señala el compromiso del profesional de la Enfermería y la esencia de su ser disciplinar, aún a pesar de los factores que intervienen en su quehacer dificultando su actuación como son las condiciones laborales, salariales y motivacionales (23); las cuales en situaciones adversas pudieron influir en la percepción del grupo de personas $(22 \%)$ 
ISSN-PRINT

1794-9831

E-ISSN 2322-7028

Vol. 16 No. 2

May - Ago 2019

Cúcuta, Colombia que no se sintieron suficientemente atendidas en esta categoría.

Para la dimensión: "mantiene relaciones de confian$z a$ ”, Molina (27) halló en su estudio que ésta se cumplió en un $79 \%$ según la consideración de sus pacientes; un comportamiento ligeramente por encima de los resultados obtenidos en el presente estudio (73\%) e igual al reportado por Ruydiaz (28) quien encontró el mismo comportamiento en el servicio de hospitalización. Este resultado también estuvo cercano a lo descubierto por Rojas y Barajas (29) en su investigación, quienes muestran para la misma dimensión un $75,92 \%$ de importancia en la percepción de los pacientes y en las valoraciones de percepción de alta de calidad. Lo reflejado en los estudios anteriores muestran un escenario prometedor, que una vez más pone de manifiesto la naturaleza de quienes ejercen la labor del cuidado profesional de la Enfermería, demostrando su característica humanizante de comunicación con el ser cuidado. Sin embargo, se evidencia también una alerta con esa proporción (27\%) significativa de pacientes que no aprobaron tal relación y que podría poner en riesgo la práctica de cuidado, lo cual merece seguir investigándose.

Con respecto al escenario de las dimensiones: " $a c$ cesibilidad", "explica y facilita" y "conforta" fue similar, observándose que la proporción de percepción de calidad por parte de los pacientes (parcial o totalmente) osciló entre $70 \%$ y $71 \%$. Una situación que difiere de lo encontrado por Chaves et al. (30) sobre esta categoría, quienes comprobaron que al sumar todos los ítems positivos para calidad se halló una percepción de accesibilidad total (100\%) de la Enfermera (o); de modo similar en lo referido a "Explica y facilita" fue de 95,6\% y en la dimensión "conforta" de $98,9 \%$ de percepción positiva de calidad del cuidado. Los datos anteriores reflejan un panorama con oportunidades para mejorar, teniendo en cuenta que las ejecución de actividades propias del Enfermero (a) se ven comprometidas por las características propias de un sistema en el que predomina la industrialización, con poca sensibilidad.

En el apartado correspondiente a la dimensión "se anticipa" muestra la percepción más baja (61\%) por parte de los pacientes a la hora de afirmar si la Enfermera (o) lo hace en las mejores condiciones (total o parcialmente); el hallazgo sugiere un comportamiento superior a lo presentado en un estudio de Rojas y
Barajas (29) donde se indica que solo el 0,52\% de los paciente perciben dicha dimensión en la categoría alta de calidad. Como se puede advertir en ambos casos la tendencia es insatisfactoria, aunque está marcada en el estudio de comparación, lo que permite percibir algunos efectos a consecuencia de la diversidad de tareas ajenas a las competencias de la Enfermería, que afectarían estos resultados.

Por otra parte, el resultado global de calidad total alcanzada sobre el cuidado de Enfermería, obtenido en la ciudad es bastante regular (62\%) según la percepción de los pacientes; en ése sentido se encontró un porcentaje importante (38\%) de susceptibilidad para mejorar, lo que sugiere la realización de una intervención que optimice la calidad de los cuidados de Enfermeros que se ofrecen; de ahí que se deba generar una discusión compartida entre academia, instituciones de salud y Enfermeras(os), al menos desde el ámbito local, utilizando el liderazgo de la Enfermería como activo en la mejora de la calidad de atención al paciente.

\section{Conclusiones}

- En el presente estudio la calidad global valorada del cuidado de Enfermería presentó una situación regular, con amplias oportunidades para mejorar.

- La dimensión "se anticipa" que indicaba la capacidad para planear con anterioridad según las necesidades del paciente y que es preventiva, fue la única percibida dentro de un rango bajo de calidad, aunque no tan alejado de la zona de mejoramiento.

- La dimensión mejor calificada en la proporción de calidad del cuidado, fue "monitorea y hace seguimiento" relacionada con los cuidados de Enfermería, los cuales implican un conocimiento propio de cada usuario y dominio de lo científico técnico; lo que indica que a pesar de los resultados regulares obtenidos en el análisis global, los conocimientos, la técnica y las habilidades son reconocidas como significativas por los usuarios atendidos.

- Existe una oportunidad de intervención para optimizar la calidad del cuidado de Enfermería encontrada, teniendo en cuenta la práctica, los recursos y la satisfacción de los pacientes con la asistencia recibida. 
- Debe valorarse continuamente la calidad del cuidado de Enfermería con el fin de cerrar brechas en dimensiones particulares y buscar tendencias positivas en la atención del paciente; también, hay que identificar factores (tanto intrínsecos como extrínsecos) que puedan influir sobre la calidad del cuidado brindado.

- Finalmente, la valoración de la calidad del cui- dado encuentra en el "Care Q" un instrumento de alta fiabilidad, que permite generar replicabilidad y trazabilidad de información que se puede mantener sistemáticamente. Este sistema favorece la monitorización a través de un Observatorio del cuidado de Enfermería, donde se pueda encontrar retroalimentación tanto para la práctica profesional como para la academia.

\section{Referencias Bibliográficas}

1. República de Colombia. Ministerio de Salud. Decreto No. 780 de 2016, mayo 6, por medio del cual se expide el Decreto Único Reglamentario del Sector Salud y Protección Social. [Internet]. Santa Fe de Bogotá: El Ministerio; 2016 [consultado 10 de septiembre de 2018]. Disponible en: https://www. minsalud.gov.co/Normatividad_Nuevo/Decreto\%200780\%20de\%202016.pdf

2. Saver BG, Martin SA, Adler RN, et al. Care that Matters: Quality Measurement and Health Care. PLoS Med 2015; 12(11):e1001902. doi:10.1371/journal.pmed.1001902

3. Kleinpell R, Kapu AN. Quality measures for nurse practitioner practice evaluation. Journal of the American Association of Nurse Practitioners 2017; 29(8):446-451. Doi: 10.1002 / 2327-6924.12474.

4. Donabedian A. La dimensión internacional de la evaluación y garantía de la calidad. Salud Pública de México. 1990 1990-03-28;32(2):5. Epub 1990-03-28.

5. Cano Sierra SM, Giraldo A, Forero C. Concepto de calidad en salud: resultado de las experiencias de la atención, Medellín, Colombia. Revista Facultad Nacional de Salud Pública. [Internet]. 2016 [Consultado 21 aug. 2018]; 34(1). Disponible en: http://aprendeenlinea.udea.edu.co/revistas/index. $\mathrm{php} /$ fnsp/article/view/19646.

6. Febré Vergara N, Mondaca Gómez K, Méndez Celis P, Badilla Morales V, Soto Parada P, et al. Quality in nursing: management, implementation and measurement. Revista Médica Clínica Las Condes. [Internet]. 2018 []; 29(3):278-287. Disponible en: https://doi.org/10.1016/j.rmclc.2018.04.008.

7. Lagoueyte Gómez MI. El cuidado de enfermería a los grupos humanos. Rev. Univ. Ind. Santander. Salud [Internet]. 2015 Aug [cited 2018 Aug 21] ; 47( 2 ): 209-213. Available from: http://www. scielo.org.co/scielo.php?script=sci_arttext\&pid $=$ S0121-08072015000200013\&lng $=$ en.

8. Luengo Martínez C, Sanhueza O. Condiciones de trabajo y su relación con la calidad del cuidado y salud del profesional de enfermería. Med. segur. trab. [Internet]. 2016; Dic [citado 2018 Sep 12]. 62(245 ): 368-380. Disponible en: http://scielo.isciii.es/scielo.php?script=sci_arttext\&pid=S0465546X2016000500008\&lng $=$ es.

9. Hernández AM, Vásquez ML. El cuidado de enfermería comprometido: Motor en la satisfacción de la gestante durante el control prenatal. Rev Univ. salud. 2015;17(1):80-96

10. Alligood MR, Tomey AM. Modelos y teorías en enfermería. Elsevier Health Sciences; 2018. 618 p.

11. Arco Canoles OC, Suarez Calle ZK. Rol de los profesionales de enfermería en el sistema de salud colombiano. Univ. Salud [Internet]. 2018 [citado 201811 de septiembre]; 20 (2): 171 - 182. Disponible en: http://www.scielo.org.co/scielo.php?script=sci_arttext\&pid=S0124-71072018000200171\&lng=en. http://dx.doi.org/10.22267/rus.182002.121.

12. Girmay A, Marye T, Haftu M, G/her D, Brhanu T, Gerensea H. Patients expectation strongly associated with patients perception to nursing care: hospital based cross sectional study. BMC Res Notes [Internet]. 2018; [citado 10 de septiembre de 2018];11. Disponible en: https://www.ncbi.nlm.nih.gov/ pmc/articles/PMC5960159 
ISSN-PRINT

$1794-9831$

E-ISSN 2322-7028

Vol. 16 No. 2

May - Ago 2019

Cúcuta, Colombia
13. Jaramillo Santiago XL, Osorio Galeano SP, Blandón Salazar DA. Quality of Nursing Care: Perception of Parents of Newborns Hospitalized in Neonatal Units. Investig Educ En Enferm [Internet]. abril de 2018 [citado 12 de septiembre de 2018];36(1). Disponible en: http://www.scielo.org.co/scielo. php?script $=$ sci abstract\&pid $=S 0120-53072018000100008 \& \operatorname{lng}=$ en\&nrm=iso\&tlng $=$ en

14. Santamaría N, García L, Sánchez Herrera B, Carrillo G. Percepción del cuidado de enfermería dado a los pacientes con cáncer hospitalizados. RLB [Internet]. 2015; [citado 17mar.2019];16(30-1):104-27. Available from: https://revistas.unimilitar.edu.co/index.php/rlbi/article/view/1443Ferreira Umpiérrez A.

15. Larson P. Percepciones del cuidado de pacientes con cáncer por enfermeras oncológicas [tesis doctoral]. San Francisco: Universidad de California; 1981.

16. Sepúlveda CG, Rojas CL, Cárdenas JO, Guevara RE, Castro HA. Estudio piloto de la validación del cuestionario CARE-Q en versión al español en población colombiana. Rev. Colomb. Enferm. 2009; 4 (4): 1-17.

17. WMA Declaration of Helsinki - Ethical Principles for Medical Research Involving Human Subjects [Internet]. 2013; [citado 21 de abril de 2014]. Disponible en: http://www.wma.net/ es/30publications/10policies/b3/

18. República de Colombia. Ministerio de Salud. Resolución No. 008430 de 1993, octubre 4, por la cual se establecen las normas científicas, técnicas y administrativas para la investigación en salud. [Internet]. Santa Fe de Bogotá. [Internet]. [citado 10 de septiembre de 2018]. Disponible en: http://www.imbiomed.com.mx/1/1/articulos.php?method=showDetail\&id_articulo=25017\&id_seccion $=1784 \&$ id ejemplar $=2583 \&$ id revista $=115$

19. Taber, K.S. The Use of Cronbach's Alpha When Developing and Reporting Research Instruments in Science Education Res Sci Educ. 2017. Disponible en https://doi.org/10.1007/s11165-016-9602-2

20. Lenis Victoria CA, Manrique Abril FG. Calidad del cuidado de enfermería percibida por pacientes hospitalizados. Aquichan [Internet]. 2015 [Consultado 21 ago. 2018]; 15(3)413-425. Disponible en: http://aquichan.unisabana.edu.co/index.php/aquichan/article/view/3925/3988.

21. Torres Contreras C, Buitrago Vera M. Percepción de la calidad del cuidado de enfermería en pacientes oncológicos hospitalizados. Rev Cuid [Internet]. 2011 Ene [citado 2018 Ago 05]; 2(1):138-148. Disponible en: http://www.scielo.org.co/scielo.php?script=sci_arttext\&pid=S221609732011000100007\&lng=es. http://dx.doi.org/10.15649/cuidarte.v2i1.49.

22. Cabrera DM. Enfermeras acusan al Gobierno, IPS y EPS de deshumanizar servicio de salud. [Artículo de prensa]. Colombia: La Fm radio; 2018 [consultado 30 de octubre de 2018]. Disponible en: https://www.lafm.com.co/salud/enfermeras-acusan-al-gobierno-ips-y-eps-de-deshumanizar-serviciode-salud

23. Boamah SA. Emergence of informal clinical leadership as a catalyst for improving patient care quality and job satisfaction. J Adv Nurs [Internet]. 2018. [consultado 30 de octubre de 2018]. Disponible en: https://doi.org/10.1111/jan.13895

24. Luengo Martínez C, Sanhueza O. Condiciones de trabajo y su relación con la calidad del cuidado y salud del profesional de enfermería. Med. segur. trab. [Internet]. 2016 Dic [citado 2019 Ene 14]; 62( 245 ): 368-380. Disponible en: http://scielo.isciii.es/scielo.php?script=sci_arttext\&pid=S0465546X2016000500008\&lng=es.

25. Molina Mula J, Vaca Auz J, Muñoz Navarro P, Cabascango Cabascango K, Cabascango Cabascango C. Gestión de la calidad de los cuidados de enfermería hospitalaria basada en la evidencia científica. Index Enferm [Internet]. 2016 Sep [citado 2018 Ago 06] ; 25( 3 ): 151-155. Disponible en: http:// scielo.isciii.es/scielo.php?script=sci_arttext\&pid=S1132-12962016000200006\&lng=es.

26. Guzmán de Melo MP. Percepción del cuidado de enfermería en un servicio de atención prenatal. Montería 2013. [Tesis en internet]. Universidad Nacional de Colombia; 2013 [citado 11 de abril de 2018]. Disponible en: http://bdigital.unal.edu.co/43080/ 
27. Molina Cardona E. Evaluación de la calidad del cuidado de enfermería en un servicio de hospitalización domiciliaria en Bogotá D.C. [Tesis en internet]. Universidad Nacional de Colombia; 2012 [citado 20 de agosto de 2018]. Disponible en: http://bdigital.unal.edu.co/6471/

28. Ruydiaz Gómez Ks, Saldarriaga Genes G, Fernández Aragón Sp. Satisfacción del usuario: un reto para la calidad del cuidado. Cienc Sal Virt. [Internet]. 2018 [Consultado 11 abril 2018]; 10(1):49-61. Disponible en: http://dx.doi.org/10.22519/21455333.969

29. Rojas Martínez W, Barajas Lizarazo MA. Percepción de la calidad del cuidado de enfermería en el servicio de urgencias. Rev Cien Cuid [Internet] 2016 [Consultado 20 agosto 2018]; 9(1):13-23. Disponible en: http://revistas.ufps.edu.co/ojs/index.php/cienciaycuidado/article/view/448.

30. Chávez D, Romero R, Zuñiga J, Romero Massa E. Percepción de la calidad del cuidado de enfermería en pacientes en el Hospital Universitario del Caribe, Cartagena [Tesis en internet]. Universidad de Cartagena; 2013 [citado 11 de abril de 2018]. Disponible en: http://190.242.62.234:8080/jspui/ handle/11227/2686 